\section{DISCUSSION}

Left atrial ball thrombus is infrequent but well recognized as a catastrophic complication of long-standing mitral valve disease and atrial fibrillation. ${ }^{1,2}$ Recent development of reliable methods for identifying the presence of left atrial ball thrombus, such as TEE, computed tomography, and magnetic resonance imaging, has contributed to early diagnosis and prompt treatment for preventing catastrophic embolic complications. However, left atrial ball thrombus not associated with mitral valve disease and atrial fibrillation is extremely rare. Left atrial enlargement secondary to a low cardiac output and high atrial filling pressure might cause thrombus formation in the left atrial cavity. In fact, left atrial thrombi that developed in patients with restrictive cardiomyopathy was previously reported. ${ }^{3,4}$ Furthermore, in patients with severe left ventricular dysfunction due to valvular or ischemic heart disease, there is a possibility of thrombus formation in the cardiac chambers. In the present patient, the left ventricular dysfunction due to severe aortic valve stenosis may have led to thrombus formation even in the presence of sinus rhythm. However, rapid growth of this ball thrombus is thought to have a connection with other factors.

The relative risk of left atrial ball thrombus in patients requiring hemodialysis for chronic renal failure remains unclear. Nishimura and his colleagues ${ }^{5}$ reported a high incidence of left atrial appendage thrombus in patients on maintenance hemodialysis. The process of hemodialysis itself is more strongly associated with thrombosis than the state of chronic renal failure because of activation of neutrophils, monocytes, and platelets from contact with the hemodialysis membrane. Therefore, it was thought that hemodialysis might affect the thrombus formation in the present patient.

This is a unique report of left atrial ball thrombus caused by severe left ventricular dysfunction due to aortic valve stenosis in the presence of sinus rhythm. Left atrial ball thrombus should be considered in patient with severe left ventricular dysfunction even though mitral valve disease or atrial fibrillation is not recognized.

\section{References}

1. Wrisley D, Giambartolomei A, Lee I, Brownlee W. Left atrial ball thrombus: review of clinical and echocardiographic manifestations with suggestions for management. Am Heart J. 1991;121:1784-90.

2. Tsioufis CP, Stefanadis CI, Tsiamis EG, Kallikazaros IE, Toutouzas PK. A free floating ball thrombus in the left atrial cavity. J Thorac Cardiovasc Surg. 1999;118:1120-2.

3. Turhan H, Ocal A, Erbay AR, Yasar AS, Cicekcioglu F, Yetkin E. Free-floating left atrial ball thrombus developed in an 11-year old child with restrictive cardiomyopathy during sinus rhythm: manifested as a major thromboembolic event. Int J Cardiol. 2005;101:111-3.

4. Arslan S, Serdar S, Gundogdu F. Fatal biatrial thrombus in a patient with idiopathic restrictive cardiomyopathy during sinus rhythm. Int $J$ Cardiol. 2007;117:e68-70.

5. Nishimura M, Hashimoto T, Kobayashi H, Fukada T, Okino K, Yamamoto N, et al The high incidence of left atrial appendage thrombosis in patients on maintenance haemodialysis. Nephrol Dial Transplant. 2003;18:2239-47.

\title{
Long-term cardiac remodeling after salvage partial left ventriculectomy in an infant with anomalous left coronary artery from the pulmonary artery
}

\author{
Stephen Westaby, PhD, MS, FETCS, FECS, FACC, ${ }^{\text {a }}$ Nick Archer, MD, ${ }^{\mathrm{b}}$ and Saul G. Myerson, MD, MRCP, FESC, ${ }^{\mathrm{c}}$ \\ Oxford, United Kingdom
}

Anomalous left coronary artery from the pulmonary artery (ALCAPA) is an unusual cause of heart failure in infancy. ${ }^{1}$ In this anomaly, left coronary blood flow is reversed with

\footnotetext{
From the Departments of Cardiothoracic Surgery, ${ }^{\mathrm{a}}$ Paediatric Cardiology, ${ }^{\mathrm{b}}$ Cardiovascular Medicine, ${ }^{\mathrm{c}}$ University of Oxford, John Radcliffe Hospital, Oxford, United Kingdom.

Received for publication Dec 13, 2007; revisions received Feb 22, 2008; accepted for publication March 23, 2008.

Address for reprints: Dr Stephen Westaby, Dept. of Cardiothoracic Surgery, John Radcliffe Hospital, Headley Way, Oxford OX3 9DU, UK. (E-mail: swestaby@ ahf.org.uk).

J Thorac Cardiovasc Surg 2009;137:757-9

$0022-5223 / \$ 36.00$

Copyright (C) 2009 by The American Association for Thoracic Surgery

doi:10.1016/j.jtcvs.2008.03.068
}

steal into the pulmonary artery. Repeated episodes of myocardial ischemia or infarction cause globally impaired left ventricular function and may substantially increase the risk of surgical correction. In this report, we provide late follow-up on an infant with ALCAPA, who following coronary reimplantation could not be separated from cardiopulmonary bypass. In the absence of circulatory support technology, radical left ventricular remodeling was undertaken with successful results.

\section{CLINICAL SUMMARY}

A 5-month-old female infant presented with heart failure manifested by breathlessness and failure to thrive. 

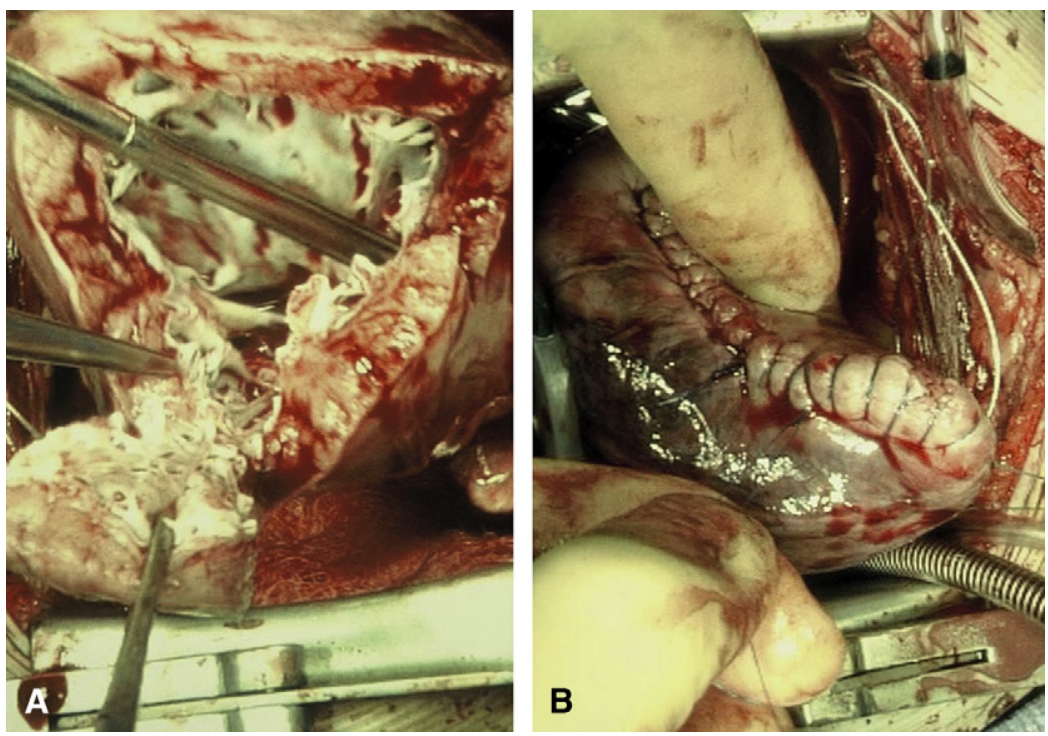

FIGURE 1. Intraoperative images of 5-month-old patient. A, Removal of infarcted myocardium in the circumflex territory. Note the white appearance of the infarcted subendocardial layer in other areas of the heart. B, External appearance once reconstructed.

Echocardiography demonstrated globally severely impaired left ventricular function (left ventricular ejection fraction $15 \%$ ) with moderate mitral regurgitation. Coronary angiography showed ALCAPA. An operation was performed to connect the left coronary ostium with the aorta using flaps derived from the pulmonary artery and aorta. These flaps extended the left main stem. ${ }^{2}$ Although cardioplegic solution was delivered to both right and left coronary arteries, myocardial contractility was poor after reperfusion and the heart could not sustain systemic blood flow. At this stage, left ventricular ejection fraction was $<10 \%$ and mitral regurgitation was severe. We did not have access to extracorporeal membrane oxygenation (ECMO) or a left ventricular assist device (LVAD). After 2 hours of supportive perfusion and several
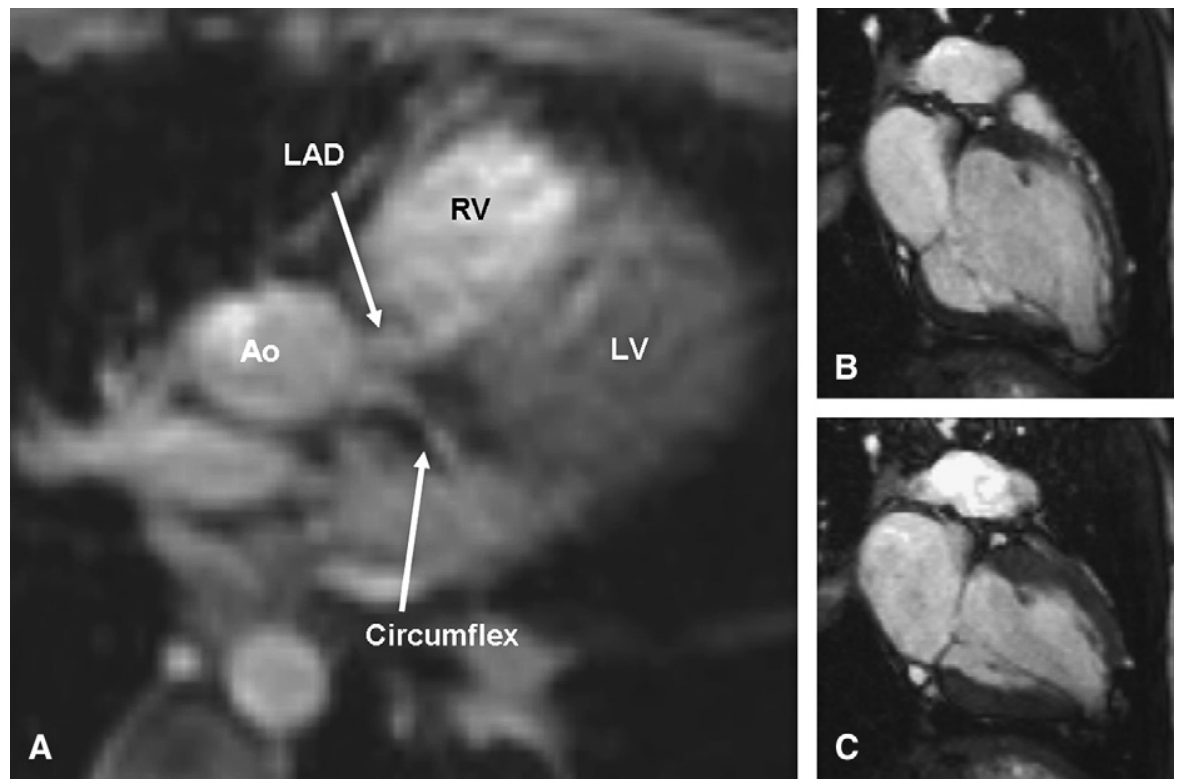

FIGURE 2. Cardiovascular magnetic resonance images of 10 -year-old patient. A, The reconstructed left coronary artery arising from the aorta. B and C, Vertical long axis (2-chamber) view demonstrating near-normal cardiac function in diastole and systole, respectively. Images are still frames from steadystate free-precession cine images with electrocardiograph gating and breath-hold on a Siemens Sonata scanner (Erlangen, Germany); field of view $340 \times$ $340 \mathrm{~mm}$; repetition time (TR) 3.08 milliseconds; (TE) echotime 1.54 milliseconds; slice thickness $6 \mathrm{~mm}$; flip angle $60^{\circ}$. Ao, Aorta; Circumflex, circumflex coronary artery; $L A D$, left anterior descending coronary artery; $L V$, left ventricle; $R V$, right ventricle. 
abortive attempts to discontinue cardiopulmonary bypass, a radical salvage procedure was attempted. There was no full-thickness dyskinetic scar, so we followed the Batista approach of partial left ventriculectomy by excising around $25 \%$ of the left ventricular circumference in the circumflex coronary territory. The myocardium itself showed circumferential endocardial fibrosis and diffuse scar formation (Figure 1,A). An Alfieri suture was applied to the mitral valve to reduce mitral regurgitation. Cardiopulmonary bypass was discontinued with inotropic support. Echocardiography then showed a much smaller, poorly compliant left ventricle but with only trivial mitral regurgitation. The sternum was closed primarily. Adrenaline infusion was weaned over the following 3 days during which amiodarone was used to suppress ventricular ectopy. The infant survived with resolution of heart failure. Histology of the excised segment confirmed a layer of subendocardial scar along with interstitial fibrosis compatible with a diagnosis of ischemic cardiomyopathy.

Now aged 10 years, she is New York Heart Association class I, participates in sport, and has normal physical and intellectual development. In view of her unrestricted lifestyle, a cardiovascular magnetic resonance scan was performed to assess left ventricular function and patency of the reconstructed left main coronary artery. The coronary had a higher than usual origin from the left lateral aspect of the aorta, but normal flow (Figure 2). The previously dilated right coronary artery had reverted to normal caliber. Left ventricular ejection fraction was $47 \%$ with normal end systolic and diastolic volume indices for her age. There was mild distal anterior hypokinesia and minimal mitral regurgitation through the dual orifice valve. The linear scar at the site of the ventricular anastomosis was the only clear sign of the myocardial resection.

\section{DISCUSSION}

There is little follow-up information regarding left ventricular morphology late after repair of ALCAPA in infancy. The purpose of this 10-year follow-up report is to suggest that the infant heart has substantial capacity for remodeling and functional improvement. Surgical and 30-day mortality are substantial for these infants. ${ }^{3}$ Because of this, the deploy- ment of ECMO or LVADs has been advocated to sustain the systemic circulation during postischemic myocardial stunning. ${ }^{4}$ We have since adopted this approach successfully in infant heart failure using both the Berlin Excor system and the Levitronix centrifugal blood pump. However, many centers still do not have ECMO or LVADs readily available, and so other salvage strategies must be considered. In patients with established transmural scar and a dyskinetic anterior wall, left ventricular aneurysmectomy has been performed.$^{5-7}$ In contrast, this child had a Batistatype partial left ventriculectomy through poorly contractile myocardium with subendocardial scar in a last attempt to sustain life. $^{2}$ Ischemic mitral regurgitation was virtually eliminated by reducing ventricular size together with the $\mathrm{Al}$ fieri suture. This contributed to the ability to separate from cardiopulmonary bypass, and mitral regurgitation remains insignificant 10 years later. The change from the globular, severely impaired left ventricle to the elliptical shape seen in Figure $1(B)$ succeeded but the very satisfactory outcome of the long-term remodeling process was unexpected. Encouraged by this result, we would again consider surgical remodeling in a life-threatening situation with or without additional circulatory support.

\section{References}

1. Arciniegas E, Farooki ZQ, Hakimi M, Green EW. Management of anomalous left coronary artery from the pulmonary artery. Circulation. 1980;62(2 Pt 2): $1180-9$.

2. Katsumata T, Westaby S. Anomalous left coronary artery from the pulmonary artery: a simple method for aortic implantation with autogenous arterial tissue. Ann Thorac Surg. 1999;68:1090-1

3. Lange R, Vogt M, Horer J, Cleuziou J, Menzel A, Holper K, et al. Long term results of repair of anomalous origin of the left coronary artery from the pulmonary artery. Ann Thorac Surg. 2007;83:1463-71.

4. del Nido PJ, Duncan BW, Mayer Jr JE, Wessel DL, LaPierre RA, Jonas RA. Left ventricular assist device improves survival in children with left ventricular dysfunction after repair of anomalous origin of the left coronary artery from the pulmonary artery. Ann Thorac Surg. 1999;67:169-72.

5. Turina M, Real F, Meier W, Senning A. Left ventricular aneurysmectomy in a 4-month-old infant. Alternative method of treatment of anomalous left coronary artery. J Thorac Cardiovasc Surg. 1974;67:915-9.

6. Carrel T, Pasic M, Turina MI. Ventricular aneurysmectomy and coronary artery ligation: an alternative method of treatment of ALCAPA syndrome. Ann Thorac Surg. 1993;55:1594-5

7. Yamagishi M, Shuntoh K, Shinkawa T, Hisaoka T, Ogawa M, Kohshi K, et al. Partial left ventriculectomy for infantile ischaemic cardiomyopathy caused by anomalous origin of the left coronary artery from the pulmonary artery. $J$ Thorac Cardiovasc Surg. 2005;130:897-9. 